\title{
Implicações da pandemia de COVID-19 para o agravamento da insegurança
}

\section{alimentar no Brasil}

\author{
Implications of the COVID-19 pandemic on the aggravation of food insecurity in Brazil
}

Implicaciones de la pandemia de COVID-19 para el agravamiento de la inseguridad alimentaria en

Brasil

Recebido: 02/09/2021 | Revisado: 09/09/2021 | Aceito: 10/09/2021 | Publicado: 12/09/2021

\author{
Lucas de Almeida Moura \\ ORCID: https://orcid.org/0000-0002-4927-4980 \\ Universidade Federal da Paraíba, Brasil \\ E-mail: moura.a.lucas@gmail.com \\ Assel Muratovna Shigayeva Ferreira \\ ORCID: https://orcid.org/0000-0002-7371-3752 \\ Universidade Federal da Paraíba, Brasil \\ E-mail: shigyassel@gmail.com \\ Igor Mikhail Marcelino Alves \\ ORCID: https://orcid.org/0000-0001-7179-3974 \\ Universidade Estadual da Paraíba, Brasil \\ E-mail: igor2018alves@gmail.com
}

\begin{abstract}
Resumo
O presente trabalho tem como objetivo analisar as implicações da pandemia de COVID-19 para o agravamento da insegurança alimentar no Brasil. Para tanto, realizou-se um estudo de revisão integrativa da literatura, cuja busca de artigos foi realizada no portal da Biblioteca Virtual em Saúde, em junho de 2020, utilizando os seguintes descritores: COVID-19; Food Insecurity; Brazil; Food; Coronavirus Infection. Foram definidos elegíveis artigos originais, com abordagem quantitativa e/ou qualitativa, realizados no Brasil, publicados de janeiro de 2020 a junho de 2021 , disponíveis gratuitamente em texto completo, nos idiomas inglês, português ou espanhol e que contemplassem a questão norteadora proposta. Os dados extraídos foram: título, autor/ano, objetivo geral, metodologia e conclusões de cada estudo. Os resultados sugerem que a pandemia de COVID-19 tem agravado a situação de insegurança alimentar e nutricional vivenciada pela população brasileira, sobretudo pelas parcelas mais vulneráveis, e isto tem sido associado a fatores diversos, mas, principalmente, a um panorama de instabilidade política e resistência a tomada de decisão, bem como aos cortes orçamentários e desconstrução de políticas e programas fundamentais para a manutenção do DHAA e da segurança alimentar e nutricional no país, os quais antecedem a pandemia e contribuem para o aumento da miséria e fome. Diante disto, apesar de serem necessários estudos de maior abrangência para melhor avaliar as implicações da pandemia de COVID-19 na insegurança alimentar da população, entretanto, é imprescindível o fortalecimento de políticas, programas e ações que atuam diretamente com o intuito de promover a segurança alimentar da população.
\end{abstract}

Palavras-chave: Segurança alimentar e nutricional; COVID-19; Políticas públicas de saúde; Pandemia por COVID19.

\begin{abstract}
The present paper aims to analyze the implications of COVID-19 pandemic on a food insecurity in Brazil. Was performed an integrative literature review with search of references from Virtual Library of Health in June 2020 with a strategy included the following terms: COVID-19; Food Insecurity; Brazil; Food; Coronavirus Infection. The references of eligible articles related to guiding question of the study with quantitative or/and qualitative approaches performed in Brazil, published in English, Spanish and Portuguese languages, in a period from January 2020 to June 2021 with open access were included to the study. Obtained data have been included: title, author/year, main objective, methodology and conclusions of each study. The results of the study suggest that COVID-19 pandemic has aggravated the situation of food and nutritional insecurity experienced by the Brazilian population, especially by its most vulnerable parts. Moreover, this situation is associated with various factors, such as political instability and resistance in a decision making in the country, accompanied by budget cuts and deconstruction of fundamental policies and programs for the maintenance of the DHAA, food and nutritional security, which precede the pandemic and contribute to the increase in poverty and hunger. Thereby, although larger studies are needed to better assess the implications of the COVID-19 pandemic on the population's food insecurity, however, it is essential to strengthen policies, programs and actions that act directly with the aim of promoting the population's food security.
\end{abstract}

Keywords: Food and nutritional security; COVID-19: Public health policies; Pandemic by COVID-19. 


\begin{abstract}
Resumen
El presente trabajo tiene como objetivo analizar las implicaciones de la pandemia de COVID-19 en el agravamiento de la inseguridad alimentaria en Brasil. Por lo tanto, se realizó un estudio de revisión integradora de la literatura, cuya búsqueda de artículos se realizó en el portal de la Biblioteca Virtual en Salud, en junio de 2020, utilizando los siguientes descriptores: COVID-19; Inseguridad Alimentaria; Brasil; Alimentación; Infección por Coronavirus. Los artículos elegibles fueron definidos como originales, con enfoque cuantitativo y/o cualitativo, realizados en Brasil, publicados entre enero de 2020 y junio de 2021, disponibles de forma gratuita en texto completo, en los idiomas inglés, portugués o español y que contemplaran la pregunta guía propuesta. Los datos extraídos fueron: título, autor/año, objetivo general, metodología y conclusiones de cada estudio. Los resultados sugieren que la pandemia de COVID-19 ha agravado la situación de inseguridad alimentaria y nutricional que vive la población brasileña, especialmente las porciones más vulnerables, y esto ha estado asociado a diversos factores, pero principalmente a un panorama de inestabilidad política y resistencia a la toma de decisiones, así como a los recortes presupuestarios y a la deconstrucción de políticas y programas fundamentales para el mantenimiento del DHAA y la seguridad alimentaria y nutricional en el país, que preceden a la pandemia y contribuyen al aumento de la miseria y el hambre. Por lo tanto, a pesar de que se necesitan estudios de mayor duración para evaluar mejor las implicaciones de la pandemia de COVID-19 en la seguridad alimentaria de la población, es imprescindible fortalecer las políticas, programas y acciones que se aplican directamente con el objetivo de promover la seguridad alimentaria de la población.
\end{abstract}

Palabras clave: Seguridad alimentaria y nutricional; COVID-19; Políticas de salud pública; Pandemia por COVID-19.

\title{
1. Introdução
}

A COVID-19 é caracterizada por um quadro de infecção respiratória, que se manifesta por meio de Síndrome Respiratória Aguda Grave (SRAG), e que possui considerável potencial de gravidade e transmissibilidade. Inicialmente, seu agente etiológico foi isolado e identificado como uma espécie de coronavírus denominada 2019-nCoV. Posteriormente a sequenciação do seu genoma e identificação da sua relação com o vírus causador do surto de SARS, ocorrido no ano de 2003, o Comitê Internacional de Taxonomia de Vírus o nomeou SARS-CoV-2 (Síndrome Respiratória Aguda Grave CoronaVírus-2) (Brasil, 2020; Santos, 2020).

Desde o relato dos primeiros casos, ainda em dezembro de 2019, em Wuhan, capital de Hubei - China, a COVID-19 passou a tomar proporção global e logo evoluiu para uma pandemia, que rapidamente alcançou números cada vez maiores de casos e, consequentemente, de óbitos decorrentes da doença. Em menos de seis meses o vírus atingiu quase todos os países do mundo (Machhi et al., 2020).

Segundo dados divulgados pela Universidade Johns Hopkins, até 1 de setembro de 2021 foram registrados aproximadamente 218 milhões de casos de COVID-19 e cerca de 4.500 milhões de óbitos em decorrência da doença em todo o mundo. Até esta data, os países com mais registros de mortes por COVID-19 eram Estados Unidos, responsável por cerca de 641 mil óbitos, Brasil com 580.5 mil óbitos e Índia, com 439 mil óbitos (Johns Hopkins University, 2020). No Brasil, de acordo com dados do Ministério da Saúde, no período de janeiro de 2020 a agosto de 2021 foram registrados, aproximadamente, 20.800 milhões de casos de COVID-19 e cerca de 580.400 mil mortes pela doença em todo o país (Brasil, 2021). Esses expressivos números colocam a Covid-19 como uma patologia de emergência mundial e, assim como os demais países afetados pela doença, o Brasil também passou a adotar medidas de distanciamento social, como a suspensão do funcionamento de órgãos públicos, do comércio, restaurantes, bares, igrejas, bem como de escolas e universidades (Menezes Morato et al., 2020).

Além disso, a pandemia de COVID-19 criou crises econômicas e de saúde pública em todo o mundo, que provavelmente testam a capacidade dos governos e legisladores nacionais, estaduais e locais de protegerem as suas populações de privação extrema por um período prolongado. Restrições nos recursos disponíveis resultantes da crise econômica mundial também aumentam o estresse envolvido nos esforços para responder a necessidade de todo tipo de assistência, principalmente alimentação (Poblacion; Segall-Corrêa; Cook; Taddei, 2021). A COVID-19 amplia as vulnerabilidades da população e, quando da soma da instabilidade no trabalho e renda das famílias com a necessária medida de isolamento social, passa a ocorrer a possibilidade de redução no acesso a alimentos e, por consequência, agravos na qualidade da alimentação e até mesmo fome. Logo, medidas 
como aquelas voltadas à garantia de renda emergencial figuram entre àquelas defendidas com prioridade para a proteção social e a promoção da segurança alimentar e nutricional no país (Jaime, 2020).

Segundo Ribeiro-Silva et al. (2020), com o advento da pandemia de COVID-19, a insegurança alimentar nos domicílios brasileiros, tanto nos níveis leve, moderado ou grave, demonstra tendência à maior magnitude. O Direito Humano a Alimentação Adequada (DHAA) está muito longe da realidade de boa parte da população mundial e, com a pandemia de COVID-19, os desafios são ainda maiores (Ribeiro-Silva et al., 2020). Além disso, mais recentemente, também houve perda de direitos sociais, afetando diretamente o acesso à alimentação e o DHAA, garantido pela Constituição Federal (Leite et al., 2020). Portanto, neste cenário pandêmico e de um mundo colapsado pela pandemia, o Brasil enfrenta questões ainda mais complexas diante de uma realidade na qual crises: sanitária, política, de seguridade social e econômica se combinam, o que torna imprescindível a realização de diagnósticos sobre o impacto desta situação na segurança alimentar da população (Galindo et al., 2021).

Diante disso, o enfrentamento a pandemia possui impactos significativos para o nível de insegurança alimentar na população e, no Brasil, mesmo antes deste cenário, já era possível observar uma tendencia de crescimento e isto tem sido agravado devido a condução da pandemia no país, que é atravessada por entraves políticos e uma desconstrução de políticas e programas fundamentais para a manutenção do DHAA e da segurança alimentar e nutricional no país. Portanto, este estudo tem como objetivo analisar as implicações da pandemia de COVID-19 para o agravamento da insegurança alimentar no Brasil.

\section{Metodologia}

Trata-se de uma revisão integrativa da literatura, na qual foi utilizado método sistematizado para síntese e análise dos trabalhos científicos disponíveis. Seguiu-se a metodologia proposta por Souza et al. (2010) que contempla os seguintes passos: definição da questão norteadora; elaboração da estratégia e execução de busca; seleção dos estudos encontrados; escolha das informações relevantes nos estudos selecionados; análise crítica dos estudos incluídos; discussão dos resultados; e apresentação da revisão integrativa.

Foi definida como questão norteadora a seguinte pergunta: "Como a pandemia de Covid-19 agrava a situação de insegurança alimentar no Brasil?”. A pesquisa foi realizada em junho de 2021 através do portal online da Biblioteca Virtual em Saúde (BVS), na qual foram utilizados os descritores combinados ao operador booleano AND: (covid-19) AND (food insecurity) AND (Brazil) AND (food) AND (coronavirus infection).

Foram definidos elegíveis os artigos originais, com abordagem quantitativa ou qualitativa, realizados no Brasil, de janeiro de 2020 a junho de 2021, disponíveis gratuitamente em texto completo, nos idiomas inglês, português ou espanhol e que contemplassem a questão norteadora proposta. A escolha do período considerou a data de reconhecimento da epidemia de Covid19 como uma situação de emergência em saúde pública no Brasil por parte do Ministério da Saúde, o que ocorreu em janeiro de 2020.

Os artigos foram selecionados conjuntamente por dois pesquisadores por meio da leitura inicial dos títulos e resumos, o que possibilitou a exclusão de trabalhos duplicados e aqueles que não se enquadravam na temática e nos critérios de elegibilidade previamente definidos. Após isto, os artigos restantes foram lidos na íntegra e incluídos no estudo. Considerando a novidade do assunto referente à pandemia de Covid-19 e as dificuldades para a realização de pesquisas científicas durante este período, que requer isolamento social, entende-se que a limitação da quantidade de trabalhos encontrados é justificada.

Os dados extraídos dos artigos selecionados incluíram: título, autor, objetivos, metodologia, resultados (caso possuíssem) e conclusões de cada estudo, sendo que os principais dados foram sistematizados e apresentados em forma de quadro na seção dos resultados. 


\section{Resultados e Discussão}

Ao todo, foram encontrados nas bases de dados 16 artigos e, destes, foram incluídos oito estudos que demonstraram satisfazer os critérios de inclusão, bem como possuírem elementos necessários para responder à questão norteadora proposta.

Figura 1. Fluxograma de seleção e inclusão dos artigos.

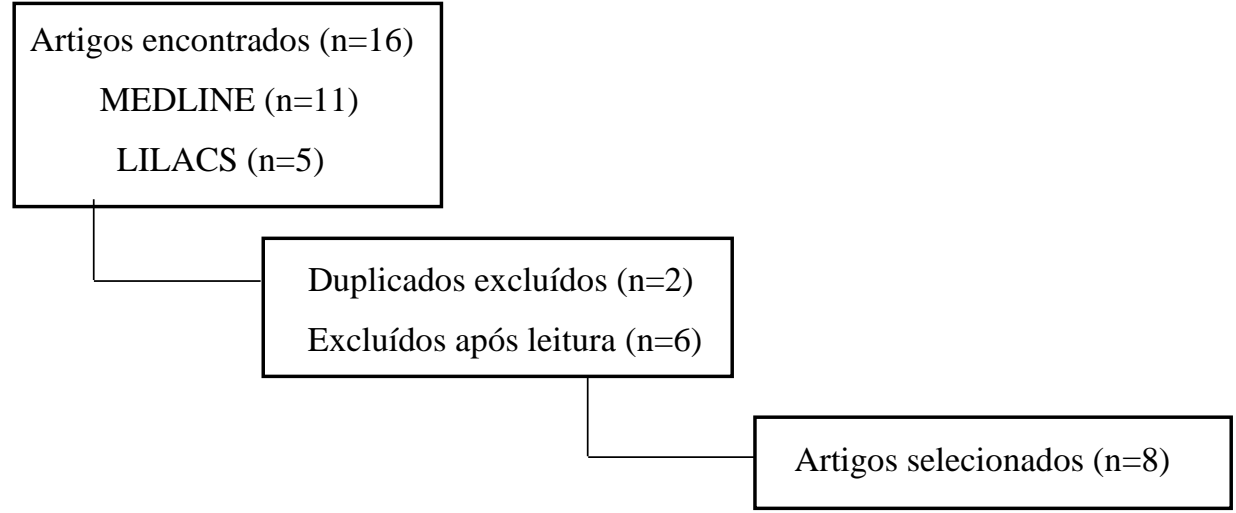

Fonte: Autores.

A maior parte dos estudos se dedicou a discutir as implicações do cenário pandêmico na insegurança alimentar no Brasil, tomando como principais métodos a aplicação da Escala Brasileira de Insegurança Alimentar (EBIA), a análise de documentos, dados e notícias acerca da pandemia de COVID-19, bem como a utilização de inquéritos epidemiológicos realizados por telefone durante um período determinado da pandemia. Além disso, diante dos resultados obtidos, alguns estudos também sugeriram estratégias a fim de contribuir para o fortalecimento das políticas de segurança alimentar e nutricional no país e garantir o direito humano à alimentação adequada e saudável.

Quadro 1. Estudos sobre insegurança alimentar e nutricional durante a pandemia de COVID-19.

\begin{tabular}{|c|c|c|c|c|}
\hline Título & Autor(es)/Ano & Objetivo geral & Metodologia & Conclusões \\
\hline $\begin{array}{lr}\text { National school feeding } \\
\text { program: } & \text { strategies to } \\
\text { overcome } & \text { food } \\
\text { insecurities } & \text { during and } \\
\text { after the } & \text { COVID-19 } \\
\text { pandemic } & \end{array}$ & $\begin{array}{l}\text { Amorim; Ribeiro Junior; } \\
\text { Bandoni (2020) }\end{array}$ & $\begin{array}{l}\text { Analisar a contribuição } \\
\text { do Programa Nacional de } \\
\text { Alimentação Escolar } \\
\text { (PNAE) para o } \\
\text { enfrentamento da fome e } \\
\text { da insegurança alimentar } \\
\text { (IA), ao propor um } \\
\text { conjunto de estratégias } \\
\text { para que o PNAE possa } \\
\text { garantir a alimentação } \\
\text { dos escolares no } \\
\text { momento de crise. }\end{array}$ & $\begin{array}{l}\text { Realizou-se um } \\
\text { diagnóstico da IA e da } \\
\text { fome no Brasil, analisou- } \\
\text { se o processo de } \\
\text { enfraquecimento das } \\
\text { políticas de segurança } \\
\text { alimentar e nutricional, } \\
\text { considerou-se a evolução } \\
\text { histórica do PNAE e sua } \\
\text { situação atual e, com } \\
\text { base nessas reflexões, } \\
\text { apresentou-se um } \\
\text { conjunto de propostas, } \\
\text { avaliando seus impactos } \\
\text { no orçamento destinado } \\
\text { ao PNAE. }\end{array}$ & $\begin{array}{l}\text { Foram sugeridas para, } \\
\text { após o retorno das aulas, } \\
\text { as seguintes estratégias: } \\
\text { levantamento da IA entre } \\
\text { os estudantes, } \\
\text { manutenção da equidade } \\
\text { da política por meio da } \\
\text { ampliação do valor do } \\
\text { repasse para municípios } \\
\text { com IDH baixo e muito } \\
\text { baixo, além de } \\
\text { atendimento dos } \\
\text { escolares de famílias em } \\
\text { IA durante as férias e o } \\
\text { recesso escolar. }\end{array}$ \\
\hline $\begin{array}{l}\text { How is Brazil facing the } \\
\text { crisis of Food and } \\
\text { Nutrition Security during } \\
\text { the } \\
\text { pandemic? }\end{array}$ & $\begin{array}{ll}\text { Carvalho; } & \text { Viola; } \\
\text { Sperandio (2020) }\end{array}$ & $\begin{array}{l}\text { Expor a situação da } \\
\text { Segurança Alimentar e } \\
\text { Nutricional (SAN) no } \\
\text { Brasil no contexto da } \\
\text { pandemia COVID-19, } \\
\text { fazendo uma análise } \\
\text { crítica desse cenário e }\end{array}$ & $\begin{array}{l}\text { Análise crítica do cenário } \\
\text { de pandemia de COVID- } \\
19 \text { e suas implicações } \\
\text { para a Segurança } \\
\text { Alimentar e Nutricional } \\
\text { neste período. Utilizou- } \\
\text { se de análise de } \\
\text { documentos oficiais, }\end{array}$ & $\begin{array}{l}\text { A pandemia COVID-19 } \\
\text { destaca a urgência de o } \\
\text { governo brasileiro voltar } \\
\text { a priorizar a agenda de } \\
\text { SAN. Isso inclui a } \\
\text { implementação } \\
\text { mecanismos de } \\
\text { garantir o Direito }\end{array}$ \\
\hline
\end{tabular}




\begin{tabular}{|c|c|c|c|c|}
\hline & & $\begin{array}{l}\text { sugerindo caminhos para } \\
\text { avançar }\end{array}$ & $\begin{array}{l}\text { dados de organizações } \\
\text { internacionais e artigos } \\
\text { científicos. }\end{array}$ & $\begin{array}{l}\text { Humano à Alimentação } \\
\text { Adequada e a expansão } \\
\text { dos programas existentes } \\
\text { de SAN. }\end{array}$ \\
\hline $\begin{array}{l}\text { Covid-19 pandemic } \\
\text { implications for food and } \\
\text { nutrition security in } \\
\text { Brazil }\end{array}$ & $\begin{array}{l}\text { Ribeiro-Silva et al. } \\
(2020)\end{array}$ & $\begin{array}{l}\text { Discutir as repercussões } \\
\text { da COVID-19 na } \\
\text { situação de segurança } \\
\text { alimentar e nutricional e } \\
\text { sua interface com } \\
\text { políticas de proteção } \\
\text { social. }\end{array}$ & $\begin{array}{lr}\text { Foram } & \text { analisados } \\
\text { documentos } & \text { oficiais } \\
\text { sobre decretos e leis } \\
\text { relativas } & \text { ao } \\
\text { enfrentamento } & \text { da } \\
\text { COVID-19 no âmbito } \\
\text { estadual e federal. }\end{array}$ & $\begin{array}{l}\text { No contexto da pandemia } \\
\text { de COVID-19, aponta-se } \\
\text { como um desafio } \\
\text { assegurar diferentes } \\
\text { mecanismos } \\
\text { contribuam para a } \\
\text { garantia do Direito } \\
\text { Humano a Alimentação } \\
\text { Adequada (DHAA), } \\
\text { potencializando } \\
\text { diferentes estratégias de } \\
\text { abastecimento alimentar. }\end{array}$ \\
\hline $\begin{array}{l}\text { Government strategies to } \\
\text { ensure the human right to } \\
\text { adequate and healthy } \\
\text { food facing the Covid-19 } \\
\text { pandemic in Brazil }\end{array}$ & Gurgel et al. (2020) & $\begin{array}{l}\text { Identificar as estratégias } \\
\text { governamentais } \\
\text { implementadas no Brasil } \\
\text { para prover o Direito } \\
\text { Humano à Alimentação } \\
\text { Adequada e Saudável em } \\
\text { contextos de elevada } \\
\text { vulnerabilidade social } \\
\text { frente à Covid-19. }\end{array}$ & $\begin{array}{l}\text { Estudo transversal, com } \\
\text { análise de documentos } \\
\text { oficiais publicados entre } \\
20 \text { de março e } 30 \text { de julho } \\
\text { de } 2020 \text { pela União, } \\
\text { Distrito Federal, estados } \\
\text { e capitais brasileiras, } \\
\text { com foco em medidas } \\
\text { que assegurem } \\
\text { disponibilidade e acesso } \\
\text { físico ou financeiro a } \\
\text { alimentos. }\end{array}$ & $\begin{array}{l}\text { Evidenciou-se que as } \\
\text { medidas governamentais } \\
\text { adotadas no Brasil não } \\
\text { são suficientes para } \\
\text { evitar a Insegurança } \\
\text { Alimentar decorrente da } \\
\text { pandemia. Observou-se } \\
\text { no país uma prevalência } \\
\text { de ações direcionadas ao } \\
\text { consumo, sem interferir } \\
\text { na cadeia produtiva. } \\
\text { Também foi evidenciada } \\
\text { a necessidade de prestar } \\
\text { assistência específica a } \\
\text { grupos em maior situação } \\
\text { de vulnerabilidade. }\end{array}$ \\
\hline $\begin{array}{l}\text { High prevalence of food } \\
\text { insecurity, the adverse } \\
\text { impact of COVID-19 in } \\
\text { Brazilian favela }\end{array}$ & Manfrinato et al. (2020) & $\begin{array}{l}\text { Investigar a prevalência } \\
\text { de insegurança alimentar } \\
\text { (IA) em duas favelas no } \\
\text { Brasil nas primeiras } \\
\text { semanas da política de } \\
\text { distanciamento social, de } \\
27 \text { de março de } 2020 \text { a } 1 \\
\text { de junho de } 2020 \text {. }\end{array}$ & $\begin{array}{l}\text { Estudo transversal } \\
\text { usando um questionário } \\
\text { online para obter } \\
\text { informações } \\
\text { características } \\
\text { socioeconômicas e } \\
\text { demográficas, os tipos de } \\
\text { lojas visitadas para } \\
\text { comprar alimentos e } \\
\text { triagem de IA. A IA foi } \\
\text { avaliada de acordo com a } \\
\text { Escala Brasileira de } \\
\text { Insegurança Alimentar. } \\
\text { A população de estudo } \\
\text { foi composta por } \\
\text { moradores de duas } \\
\text { favelas localizadas na } \\
\text { cidade de São Paulo -SP. }\end{array}$ & $\begin{array}{l}\text { Metade dos participantes } \\
\text { experimentou } \\
\text { moderada ou } \text { grave e } \\
\text { quase } 10 \% \text { sentiram } \\
\text { fome. Os dados } \\
\text { querem } \\
\text { crianças correm um risco } \\
\text { menor de IA moderada a } \\
\text { grave. É possível que } \\
\text { programas sociais } \\
\text { estabelecidos } \\
\text { nacionalmente, como } \\
\text { o Bolsa } \\
\text { Família, estivessem } \\
\text { protegendo } \\
\text { famílias. }\end{array}$ \\
\hline $\begin{array}{l}\text { Coronavirus disease } \\
\text { 2019, food security and } \\
\text { maternal mental health in } \\
\text { Ceará, Brazil: a repeated } \\
\text { cross-sectional survey }\end{array}$ & Rocha et al. (2021) & $\begin{array}{l}\text { Quantificar a mudança } \\
\text { no risco de insegurança } \\
\text { alimentar e transtorno } \\
\text { mental materno (DMM) } \\
\text { antes e durante a } \\
\text { pandemia de doença } \\
\text { coronavírus } \\
\text { (COVID-19). }\end{array}$ & $\begin{array}{l}\text { Estudo transversal, } \\
\text { realizado entre julho e } \\
\text { setembro de } 2020 \text {, com } \\
\text { mães e filhos inscritos } \\
\text { em uma pesquisa de base } \\
\text { populacional de } 2017 . \\
\text { Para a avaliação da } \\
\text { segurança alimentar foi } \\
\text { utilizada a Escala } \\
\text { Brasileira de Segurança } \\
\text { Alimentar (EBIA). }\end{array}$ & $\begin{array}{l}\text { O risco de insegurança } \\
\text { alimentar e DMM no } \\
\text { Ceará aumentou durante } \\
\text { a pandemia de COVID- } \\
\text { 19. Esses resultados } \\
\text { destacam a necessidade } \\
\text { de políticas e } \\
\text { intervenções para reduzir } \\
\text { o impacto da pandemia } \\
\text { COVID-19 na saúde } \\
\text { materno-infantil, } \\
\text { nutrição e bem-estar no } \\
\text { Brasil. }\end{array}$ \\
\hline
\end{tabular}




\begin{tabular}{|c|c|c|c|c|}
\hline $\begin{array}{l}\text { Unemployment, poverty, } \\
\text { and hunger in Brazil in } \\
\text { Covid-19 pandemic } \\
\text { times }\end{array}$ & Neves et al. (2021) & $\begin{array}{l}\text { Apresentar } \\
\text { desdobramentos } \\
\text { preliminares da } \\
\text { pandemia de COVID-19 } \\
\text { sobre o desemprego, a } \\
\text { pobreza e a fome no } \\
\text { Brasil. }\end{array}$ & $\begin{array}{l}\text { Utilizaram-se dados } \\
\text { sobre a taxa de } \\
\text { desocupação, } \\
\text { solicitações de seguro- } \\
\text { desemprego } \\
\text { contingente de famílias } \\
\text { em extrema pobreza e em } \\
\text { insegurança alimentar, } \\
\text { coletados em sistemas de } \\
\text { informação } \\
\text { governamentais, em } \\
\text { pesquisas publicadas por } \\
\text { órgãos públicos, em } \\
\text { artigos científicos e em } \\
\text { portais de notícias. }\end{array}$ & $\begin{array}{l}\text { A pandemia de COVID- } \\
19 \text { consiste em um novo } \\
\text { elemento potencializador } \\
\text { do aumento recente da } \\
\text { fome no Brasil, que } \\
\text { ocorre paralelamente ao } \\
\text { desmonte dos programas } \\
\text { de Segurança Alimentar } \\
\text { e Nutricional e à } \\
\text { ampliação de medidas de } \\
\text { austeridade fiscal } \\
\text { iniciadas com a crise } \\
\text { político-econômica em } \\
\text { 2015. }\end{array}$ \\
\hline $\begin{array}{l}\text { Trends and inequalities } \\
\text { in food insecurity during } \\
\text { the } \quad \text { COVID-19 } \\
\text { pandemic: results of four } \\
\text { serial epidemiological } \\
\text { surveys }\end{array}$ & Santos et al. (2021) & $\begin{array}{l}\text { Analisar tendências e } \\
\text { desigualdades na } \\
\text { prevalência de } \\
\text { insegurança alimentar na } \\
\text { pandemia de COVID-19, } \\
\text { de acordo com fatores } \\
\text { sociodemográficos e com } \\
\text { medidas de } \\
\text { distanciamento social. }\end{array}$ & $\begin{array}{l}\text { Dados de quatro } \\
\text { inquéritos } \\
\text { epidemiológicos seriados } \\
\text { sobre a COVID-19 } \\
\text { desenvolvidos entre maio } \\
\text { e junho de 2020, com } \\
\text { adultos e idosos } \\
\text { residentes na cidade de } \\
\text { Bagé, Rio Grande do Sul, } \\
\text { Brasil. Insegurança } \\
\text { alimentar foi avaliada por } \\
\text { meio da versão curta } \\
\text { da Escala Brasileira de } \\
\text { Insegurança } \\
\text { Alimentar (EBIA), como o } \\
\text { período recordatório } \\
\text { adaptado ao início das } \\
\text { medidas de } \\
\text { distanciamento social no } \\
\text { município. }\end{array}$ & $\begin{array}{l}\text { Evidenciou-se forte } \\
\text { associação } \\
\text { insegurança alimentar } \\
\text { com os aspectos } \\
\text { sociodemográficos dos } \\
\text { entrevistados, o que pode } \\
\text { indicar o potencial } \\
\text { impacto econômico da } \\
\text { pandemia na situação } \\
\text { alimentar dos domicílios. }\end{array}$ \\
\hline
\end{tabular}

Fonte: Autores (2021).

Em relação aos resultados, de modo geral, verifica-se que a pandemia de COVID-19 tem aprofundado e tornado cada vez mais grave a situação da insegurança alimentar (IA) no Brasil. Durante este período, que ainda perdura ao passo que a doença permanece vitimando a população, tem sido constatado um aumento da IA, principalmente nas parcelas mais vulneráveis, nas quais é possível constatar algum nível de IA mesmo antes da pandemia e, com a crítica situação de saúde pública, o cenário se agrava ainda mais (Manfrinato et al., 2020; Rocha et al., 2021).

O estudo realizado por Manfrinato et al. (2020) analisou a prevalência de insegurança alimentar (IA) em duas favelas no Brasil nas primeiras semanas da política de distanciamento social. Verificou-se que, entre os meses de março a junho de 2020 , período que representa um intenso crescimento da curva de casos e óbitos por COVID-19 no Brasil, a IA (forma moderada a grave) foi experimentada por quase metade dos indivíduos entrevistados e, cerca de $10 \%$ destes, relataram sentir fome durante esse período. Do mesmo modo, Rocha et al. (2021), com o objetivo de quantificar a mudança no risco de insegurança alimentar e transtorno mental materno (DMM) antes e durante a pandemia de COVID-19 no estado do Ceará, constataram que o risco de IA aumentou durante o período considerado. Os autores destacaram a necessidade de políticas e intervenções para redução do impacto da pandemia de COVID-19 na nutrição no Brasil.

Os impactos da COVID-19 sobre a segurança alimentar e nutricional são diversos, pois são resultantes de elementos que se relacionam com fatores de risco que têm sido identificados e associados a um pior prognóstico clínico da infecção pelo SARS-CoV-2, há exemplo da obesidade e outras doenças crônicas, bem como a má nutrição, que se apresenta de diferentes formas, e que é agravada no contexto da pandemia (Jaime, 2020). 
De acordo com o relatório "O Estado da Insegurança Alimentar e Nutrição no mundo" (SOFI 2021), produzido pela Food and Agriculture Organization (FAO) junto à Organização das Nações Unidas (ONU), a fome mundial passou por um dramático agravamento em 2020 e, provavelmente, este aumento está associado às consequências da pandemia de COVID-19. O relatório estima que cerca de 811 milhões de pessoas em todo o mundo estavam subalimentadas no ano de 2020, o que sugere, segundo a Organização, a necessidade de um grande esforço mundial para honrar com a promessa de superação da fome até 2030.

Nesse sentido, a atuação dos governos frente a situação de calamidade posta pela pandemia de COVID-19 se torna preponderante para a redução desta problemática. Entretanto, se por um lado, no Brasil, programas sociais e de transferência de renda estabelecidos nacionalmente, como o Programa Bolsa Família, contribuem para a proteção de famílias de menores quanto a presença da IA, por outro, o desmonte dos programas de Segurança Alimentar e Nutricional e a ampliação de medidas de austeridade fiscal iniciadas com a crise político-econômica em 2015 potencializam a identificação de diferentes níveis de IA na população e a pandemia de COVID-19 se torna um elemento que concorre em paralelo com esta situação, agravando a situação da fome no país (Santos et al., 2021).

Ribeiro-Silva et al. (2020) apontam como desafio assegurar diferentes mecanismos que contribuam para a garantia do Direito Humano a Alimentação Adequada (DHAA). Segundo estudo conduzido por Gurgel et al. (2020), no qual se buscou identificar as estratégias governamentais implementadas no Brasil para prover o Direito Humano à Alimentação Adequada e Saudável no contexto de elevada vulnerabilidade social frente à COVID-19, os autores chamam atenção para a constatação de que as medidas governamentais adotadas no Brasil não têm sido suficientes para evitar a IA decorrente da pandemia. Conforme o estudo, observou-se no país uma prevalência de ações direcionadas ao consumo, sem interferir na cadeia produtiva. Além disso, também foi evidenciada a necessidade de prestar assistência específica a grupos em maior situação de vulnerabilidade.

Ao analisar tendências e desigualdades na prevalência de insegurança alimentar na pandemia de COVID-19, de acordo com fatores sociodemográficos e com medidas de distanciamento social, Santos et al. (2021) constataram que a insegurança alimentar possui forte associação com os aspectos sociodemográficos dos entrevistados, o que pode indicar o potencial impacto econômico da pandemia na situação alimentar dos domicílios. Segundo Patrícia Constante Jaime (2020), a renda familiar está associada à segurança alimentar e nutricional e a adoção de políticas econômicas e sociais voltadas para o aumento da renda de famílias brasileiras resultaram em redução da desnutrição infantil e da fome, no entanto, a autora chama a atenção para o fato de que a pandemia de COVID-19 potencializa as vulnerabilidades de mais de $25 \%$ da população que vive em situação de pobreza e extrema pobreza, conforme dados de 2018 do IBGE e, considerando as medidas de austeridade fiscal mencionadas anteriormente, este número tende a ser ainda maior.

Nessa perspectiva, o enfraquecimento das políticas de Segurança Alimentar e Nutricional é um agravante significativo para o Brasil e que, nos últimos anos recentes, pode ser ilustrado pela desarticulação do Sistema Nacional de Segurança Alimentar (SISAN) e extinção, mediante medida provisória assinada pelo presidente Jair Bolsonaro, do Conselho Nacional de Segurança Alimentar e Nutricional (CONSEA) e da Câmara Interministerial de Segurança Alimentar e Nutricional (CAISAN); pela não realização da Conferência Nacional de Segurança Alimentar e Nutricional e não elaboração do plano previsto para o período 2020-2023; bem como pela redução no orçamento e número de beneficiários do Programa Bolsa Família (Amorim; Ribeiro Junior; Bandoni, 2020).

Amorim, Ribeiro Junior e Bandoni (2020) destacam ainda, dentre este processo de enfraquecimento, os demais cortes orçamentários que atingem outros importantes programas, tal como o Programa de Aquisição de Alimentos da Agricultura Familiar (PAA) e, também, a redução dos estoques estratégicos de alimentos da Companhia Nacional de Abastecimento (CONAB), que são fundamentais para a manutenção de uma política de garantia de preços mínimos e extremamente úteis em situações de crise, tal qual a pandemia de COVID-19, pois podem suprir escolas e a necessidade de assistência alimentar da 
população. O Programa Nacional de Alimentação Escolar (PNAE) também é outro importante programa impactado pelo enfraquecimento da agenda de SAN no Brasil e pela situação imposta pela pandemia de COVID-19. De modo geral, sua execução passou a consistir, durante o período de suspensão das aulas em razão de situação de emergência ou calamidade pública, na distribuição de gêneros alimentícios adquiridos com recursos do PNAE aos pais ou responsáveis dos estudantes das escolas públicas da educação básica, o que fez surgir preocupações quanto ao caráter restritivo dos critérios de elegibilidade para o recebimento de alimentos ou refeições, bem como acerca da manutenção da aquisição dos alimentos da agricultura familiar e a qualidade da alimentação fornecida.

Para Carvalho, Viola e Sperandio (2020) a pandemia de COVID-19 ressalta a urgência de o governo brasileiro voltar a ter como uma de suas prioridades a agenda de SAN, o que inclui a expansão dos programas existentes de SAN e a implementação de mecanismos para garantir o Direito Humano à Alimentação Adequada. De acordo com o estudo "Efeitos da pandemia na alimentação e na situação da segurança alimentar no Brasil", realizado por Galindo et al. (2021), 59\% dos domicílios entrevistados demonstraram estar em situação de insegurança alimentar durante a pandemia e uma parte significativa deles diminuiu o consumo de alimentos imprescindíveis para a dieta regular da população (redução de $44 \%$ no consumo de carnes e de $41 \%$ no consumo de frutas).

Os autores enfatizam que, mesmo antes da pandemia de COVID-19 o Brasil já vinha demonstrando uma tendência de diminuição da segurança alimentar da população, conforme dados da Pesquisa de Orçamento Familiar 2017-2018, realizada pelo Instituto Brasileiro de Geografia e Estatística (IBGE), que revelou que a situação de segurança alimentar era vivenciada por 63,3\% dos domicílios pesquisados, uma redução de 14,1\% em comparação aos resultados da Pesquisa Nacional por Amostra de Domicílios de 2013 (PNAD - 2013), que havia registrado o melhor nível de segurança alimentar de toda sua série histórica $(77,4 \%)$.

Em nota técnica sobre alimentação e segurança alimentar como pauta para as eleições de 2022, publicada pela Rede Brasileira de Mulheres Cientistas, as pesquisadoras chamam atenção para os dados alarmantes da situação de insegurança alimentar no Brasil e como esse fato deve ser objeto de preocupação dos futuros parlamentares e representantes do executivo estadual e federal (Grisa et al., 2021). Também, em relatório publicado pela Organização pelo Direito Humana à Alimentação e à Nutrição Adequadas (FIAN Brasil), intitulado "O DHANA e a COVID-19 - o direito à alimentação e nutrição adequadas no contexto da pandemia", discute-se como a crise sanitária e as decisões tomadas para o seu enfrentamento ampliaram o desemprego, inflacionaram a comida, agravaram as vulnerabilidades históricas e impulsionaram a fome (Castro, 2021).

As várias crises que se combinam no Brasil afunilam ainda mais a situação do impacto da pandemia de COVID-19 para a qualidade de vida da população durante esse período e, consequentemente, para a garantia da segurança alimentar. Fatores como divergências entre os governos federal e estaduais/municipais, bem como a resistência na adoção de medidas de controle da circulação de pessoas parecem ter contribuído significativamente para o trágico número de casos e óbitos registrados pelo país que, até 30 de agosto de 2021, somavam 20.752.281 milhões de casos e 579.574 mil óbitos, segundo dados do painel de monitoramento do Conselho Nacional de Secretários de Saúde (CONASS, 2021). Desde os primeiros meses da pandemia, tem sido chamada a atenção para o fato de a condição de insegurança alimentar, já instalada, poderia ser acelerada pelas consequências da rápida propagação do SARS-CoV-2 (Oliveira; Abranches; Lana, 2020) e, diante da emergência de novas variantes e uma baixa cobertura vacinal da população, surge uma preocupação ainda maior sobre o panorama futuro da IA no país, pois, como visto, a agenda de SAN não tem sido uma prioridade no país nos últimos anos. 


\section{Conclusão}

A situação da insegurança alimentar no Brasil já vinha sendo motivo de preocupação mesmo antes da eclosão da pandemia de COVID-19 e, com a circulação do vírus e as consequências da transmissão e adoecimento em massa da população, o cenário tornou ainda mais grave um problema que já requisitava atenção.

O panorama de emergência em saúde pública tem implicado no agravamento da insegurança alimentar, o que segue uma tendencia do comportamento mundial, uma vez que a pandemia requisita ações específicas e uma atuação ostensiva por parte dos governantes a fim de assistir a população na garantia do direito humano à alimentação adequada. No entanto, a situação do Brasil é agravada tanto pela crise sanitária, quanto por crises políticas e econômicas que antecedem a chegada oficial do vírus ao país. Além disso, a não priorização das políticas e programas de manutenção da segurança alimentar e nutricional da população é um fator que se soma a tudo isto.

Portanto, apesar de serem necessários estudos de maior abrangência para melhor avaliar as implicações da pandemia de COVID-19 na insegurança alimentar da população, é imprescindível o fortalecimento de políticas, programas e ações que atuam diretamente com o intuito de promover a segurança alimentar da população.

\section{Referências}

Alpino, T. M. A., Santos, C. R. B., Barros, D. C., \& Freitas, C. M. (2020). Cadernos de Saúde Pública, 36(8):e00161320, 1-17.

Amorim, A. L. B., Ribeiro Junior, J. R. S., \& Bandoni, D. H. (2020). National school feeding program: strategies to overcome food insecurities during and after the COVID-19 pandemic. Revista de Administração Pública, 54(4), 1134-1145.

Brasil. Ministério da Saúde. (2021). Painel Coronavírus Brasil. https://covid.saude.gov.br/

BVS. (2021). Biblioteca virtual em saúde - Ministério da Saúde. https://bvsms.saude.gov.br/

Carvalho, C. B., Viola, P. C. A. F., \& Sperandio, N. (2020). How is Brazil facing the crisis of Food and Nutrition Security during the COVID-19 pandemic? Public Health Nutrition, 24(3), 561-564.

Castro, T. P. (2021). Informe: O DHANA e a COVID-19: o direito humano à alimentação e à nutrição adequadas no contexto da pandemia. FIAN.

CONASS. Conselho Nacional de Secretários de Saúde. (2021). Painel CONASS COVID-19. https://www.conass.org.br/painelconasscovid19/

FAO, IFAD, UNICEF, WFP, WHO. (2021). The State of Food Security and Nutrition in the World 2021. Transforming food systems for food security, improved nutrition and affordable healthy diets for all. FAO, 1-240.

Galindo, E., Teixeira, M. C., Araújo, M., Motta, R., Pessoa, M., Mendes, L., \& Rennó, L. (2021). "Efeitos da pandemia na alimentação e na situação da segurança alimentar no Brasil". Food for Justice Working Paper Series, 4, 1-41.

Grisa, C., Rozendo, C., Santana, L., Rigon, S., \& Zimmermann, S. (2021). Nota Técnica n 13 - Alimentação e segurança alimentar como pauta para as eleições de 2022. Rede Brasileira Mulheres Cientistas, 1-7. https://mulherescientistas.org/wp-content/uploads/2021/08/NT-13.pdf

Gurgel, A. M., Santos, C. C. S., Alves, K. P. S., Araújo, J. M., \& Leal, V. S. (2020). Government strategies to ensure the human right to adequate and healthy food facing the Covid-19 pandemic in Brazil. Ciência \& Saúde Coletiva, 25(12), 4945-4956.

Jaime, P. C. (2020). Pandemia de COVID-19: implicações para a (in)segurança alimentar e nutricional. Ciência e Saúde Coletiva, 25 (7), 2504.

Johns Hopkins University. (2021). Coronavirus Resource Center. https://coronavirus.jhu.edu/.

Leite, M. S., Ferreira, A. A., Bresan, D., Araújo, J. R., Tavares, I. N., \& Santos, R. V. (2020). Indigenous protagonism in the context of food insecurity in times of COVID-19. Rev. Nutr., 33:e200171, 1-6.

Machhi, J., Herskovitz, J., Senan, A. M., Dutta, D. et al. (2020). The natural history, pathobiology and clinical manifestations of SARS-CoV-2 infections. Journal of Neuroimmune Pharmacology, 15(3), 359-386.

Manfrinato, C. V., Marino, A., Condé, V. F., Franco, M. C. P., Stedefeldt, E., \& Tomita, L. Y. (2020). High prevalence of food insecurity, the adverse impact of COVID-19 in Brazilian favela. Public Health Nutrition, 24(6), 1210-1215.

Neves, J. A., Machado, M. L., Oliveira, L. D. A., Moreno, Y. M. F., Medeiros, M. A. T., \& Vasconcelos, F. A. G. (2021). Unemployment, poverty, and hunger in Brazil in Covid-19 pandemic times. Rev. Nutr., 34:e200170, 1-7.

Oliveira, T. C., Abranches, M. V., \& Lana, R. M. (2020). (In)segurança alimentar no contexto da pandemia por SARS-CoV-2. Cadernos de Saúde Pública, 36(4):e00055220, 1-6. 
Research, Society and Development, v. 10, n. 12, e30101220150, 2021

(CC BY 4.0) | ISSN 2525-3409 | DOI: http://dx.doi.org/10.33448/rsd-v10i12.20150

Poblacion, A., Segall-Corrêa, A. M., Cook, J., \& Taddei, J. A. A. C. (2021). Validade de um instrumento de triagem com dois itens para identificar famílias em risco de insegurança alimentar no Brasil. Cadernos de Saúde Pública, 37(6):e00132320, 1-12.

Ribeiro-Silva, R. C., Pereira, M., Campello, T., Aragão, E., Guimarães, J. M. M., Ferreira, A. J. F., Barreto, M. L., \& Santos, S. M. C. (2020). Covid-19 pandemic implications for food and nutrition security in Brazil. Ciência \& Saúde Coletiva, 25(9), 3421-3430.

Rocha, H. A. L., Sudfeld, C. R., Leite, A. J. M., Rocha, S. G. M. O., Machado, M. M. T., Campos, J. S., Silva, A. C., \& Correia, L. L. (2021). Coronavirus disease 2019, food security and maternal mental health in Ceará, Brazil: a repeated cross-sectional survey. Public Health Nutrition, 24(7), 1836-1840.

Santos, L. P., Schäfer, A. A., Meller, F. O., Harter, J., Nunes, B. P., Silva, I. C. M., \& Pellegrini, D. C. P. (2021). Trends and inequalities in food insecurity during the COVID-19 pandemic: results of four serial epidemiological surveys. Cadernos de Saúde Pública, 37(5):e00268520, 1-14.

Souza, M. T., Silva, M. D., \& Carvalho, R. (2010). Revisão integrativa: o que é e como fazer. Einstein, 8, (1), 102-106. 